Published in final edited form as:

Qual Life Res. 2017 September ; 26(9): 2333-2349. doi:10.1007/s11136-017-1571-z.

\title{
Impact of an electronic monitoring device and behavioral feedback on adherence to multiple sclerosis therapies in youth: results of a randomized trial
}

\author{
E. Ann Yeh ${ }^{1,2}$, Stephanie A. Grover ${ }^{1}$, Victoria E. Powell ${ }^{3}$, Gulay Alper ${ }^{4}$, Brenda L. Banwell ${ }^{5}$, \\ Kim Edwards ${ }^{6}$, Mark Gorman ${ }^{7}$, Jennifer Graves ${ }^{8}$, Timothy E. Lotze ${ }^{9}$, Jean K. Mah ${ }^{10}$, Lauren \\ Mednick $^{7}$, Jayne Ness ${ }^{11}$, Maya Obadia ${ }^{12,13}$, Ruth Slater ${ }^{6}$, Amy Waldman ${ }^{5}$, Emmanuelle \\ Waubant $^{8}$, and Carolyn E. Schwartz ${ }^{3,14}$ on behalf of the Pediatric MS Adherence Study \\ Group \\ ${ }^{1}$ Pediatric MS and Neuroinflammatory Disorders Program, Division of Neurology, Department of \\ Pediatrics, Neuroscience and Mental Health, Hospital for Sick Children Research Institute, \\ Hospital for Sick Children, 555 University Avenue, Rm 6D33, Toronto, ON M5G1X8, Canada \\ ${ }^{2}$ Faculty of Medicine, The University of Toronto, 1 King's College Circle \#3172, Toronto, ON M5S \\ 1A8, Canada \\ ${ }^{3}$ DeltaQuest Foundation Inc., 31 Mitchell Road, Concord, MA 01742, USA \\ ${ }^{4}$ Children's Hospital of Pittsburgh, University of Pittsburgh School of Medicine, 4401 Penn \\ Avenue, Pittsburgh, PA 15224, USA \\ ${ }^{5}$ Division of Neurology, Children's Hospital of Philadelphia, 3401 Civic Center Blvd., Philadelphia, \\ PA 19104, USA \\ ${ }^{6}$ Department of Psychiatry, The Hospital for Sick Children, 555 University Avenue, Toronto, ON \\ M5G1X8, Canada
}

\footnotetext{
Correspondence to: E. Ann Yeh.

Availability of data and supporting materials Supporting documentation for our findings is provided in manuscript data and figures and tables. Scientists wishing to gain access to our data may contact the first author (EAY), who will consider such requests on a caseby-case basis, subject to the scientific rigor of the proposed research question.

Compliance with ethical standards

Conflict of interest The authors (SG, VEP, GA, KE, MG, TEM, JM, LM, JN, MO, RS, EW and CES) have no relevant conflicts of interest to disclose. EAY and CES wrote the first draft of the manuscript and neither received an honorarium, grant, or other form of payment to do so. BLB serves as a consultant to Novartis for the purposes of a clinical trial and as an unpaid advisor to Biogen, Teva neuroscience and Sanofi. She is also a chief editor for Multiple Sclerosis and Related Disorders and is on the editorial board for Neurology. JG has received grant funding from the Race to Erase MS, Biogen and Genentech. AW has received grant funding from the National Institutes of Health (USA) and Biogen Idec. EAY receives research funding from NMSS, CMSC, OIRM, SCN, CBMH Chase an Idea, SickKids Foundation, Rare Diseases Foundation, MS Scientific Foundation (Canada), McLaughlin Centre, Mario Batalli Foundation. She performs relapse adjudication for ACI, has received unrestricted funding for a symposium from the Guthy Jackson Charitable Foundation and Teva and has served on a Scientific Advisory Board for Neurotoxicity with Juno Pharmaceuticals. Ethical approval All procedures performed in this study involving human participants were in accordance with the ethical standards of the institutional and/or national research committee and with the 1964 Helsinki Declaration and its later amendments or comparable ethical standards.

Informed consent Informed consent was obtained from all individual participants and their parent or legal guardian included in this study.

Study Sites We are also grateful for the hard work and dedication of the investigators and study teams at each site (Table 7).
} 
${ }^{7}$ Boston Children's Hospital, Harvard Medical School, 300 Longwood Avenue, Boston, MA 02115, USA

8University of California San Francisco, 505 Parnassus Avenue, San Francisco, CA 94143, USA

${ }^{9}$ Texas Children's Hospital, Baylor College of Medicine, 6621 Fannin Street, Houston, TX 77030, USA

${ }^{10}$ Alberta Children's Hospital, 2888 Shanganappi Trail NW, Calgary, AB T3B 6A8, Canada

${ }^{11}$ University of Alabama at Birmingham, 1720 2nd Avenue, Birmingham, AL 35294, USA

${ }^{12}$ ELLICSR: Health, Wellness, and Cancer Survivorship Centre, University Health Network, 585

University Avenue, Toronto, ON M5G 2C4, Canada

${ }^{13}$ Department of Psychology, Faculty of Medicine, University of Toronto, 1 King's College Circle \#3172, Toronto, ON M5S 1A8, Canada

${ }^{14}$ Departments of Medicine and Orthopaedic Surgery, Tufts University Medical School, 800 Washington Street, Boston, MA 02111, USA

\section{Abstract}

Purpose-To report the results of a randomized controlled trial using an electronic monitoring device (EM) plus a motivational interviewing (MI) intervention to enhance adherence to diseasemodifying therapies (DMT) in pediatric MS.

Methods-Fifty-two youth with MS (16.03 \pm 2.2 years) were randomized to receive either MI ( $n$ $=25$ ) (target intervention) or a MS medication video ( $n=27)$ (attention control). Primary endpoint was change in adherence. Secondary outcomes included changes in quality of life, well-being and self-efficacy. Random effects modeling and Cohen's effect size computation evaluated intervention impact.

Results-Longitudinal random effect models revealed that the MI group decreased their EM adherence (GroupxTime interaction $=-0.19$ ), while increasing frequency of parental DMT reminder (26.01)/administration (11.69). We found decreased EM use in the MI group at 6 months (Cohen's $d=-0.61)$, but increased pharmacy refill adherence $(d=0.23)$. Parental reminders about medication increased in MI subjects vs controls ( $d=0.59$ at 3 months; $d=0.70$ at 6 months). We found increases in self-reported adherence $(d=0.21)$ at 3 but not 6 months, fewer barriers to adherence at three $(d=-0.58)$ and six months $(d=-0.31)$, better physical $(d=0.23$ at 3 months; $d$ $=0.45$ at 6 months), emotional ( $d=0.25$ at 3 months) and self-efficacy function ( $d=0.55$ at 3 months; 0.48 at 6 months), but worse well-being, including self-acceptance ( $d=-0.53$ at 6 months) and environmental mastery ( $d=-0.42$ at 3 and 6 months) in intervention as compared to control patients.

Conclusions-Participants receiving MI + EM experienced worsening on objective measures of adherence and increased parental involvement, but improved on some self- and parent-reported measures. MI participants reported improvements in quality of life and self-efficacy, but worsened well-being. 


\section{Keywords}

Multiple sclerosis; Pediatric; Quality of life; Well-being; Behavioral intervention

\section{Introduction}

Disease-modifying therapies (DMTs) are being used widely for children and adolescents with MS [1]. Literature regarding medication adherence in pediatric MS is limited, but two studies have suggested poor medication adherence in up to $70 \%$ of this population [1-3]. Adolescence poses specific challenges with regard to medication adherence due to an increase in risk-taking behaviors, greater reliance on peers and growing independence.

Interventions for medication adherence that have been studied may be grouped into (1) patient education; (2) changes in medication administration, i.e., improving dosing schedules; (3) improving physician/patient communication; (4) improving access to care; and (5) behavioral/family interventions. Of these, behavioral and multi-component interventions are most effective, with one meta-analysis of psychological approaches to promoting adherence in pediatric chronic conditions showing a medium effect size for behavioral, but only a small effect size for educational interventions [4]. A subsequent metaanalysis using a different methodology found similar effect sizes for multifaceted and behavioral interventions and negligible effect sizes for purely technological interventions, such as multimedia computer programs or video games $[5,6]$. All of these studies have been small and have shown only small to medium effects.

Effective, multi-component interventions that include cognitive-behavioral approaches are costly and time-consuming to administer, as they involve individual visits with a therapist on a weekly basis. They may not suit patients who must travel long distances for care. Conversely, behavioral approaches, which focus on behavioral change using specific interview techniques, are simple to administer, and their efficacy is straightforward to evaluate. Among these techniques, motivational interviewing (MI) has been widely used to encourage self-directed motivation for behavioral change. MI uses interview techniques focused on open-ended questions, collaboration and reflective questioning. It has been shown to be effective in promoting lifestyle change [7]. The effectiveness of this approach may be augmented by the use of an electronic monitoring (EM) device, such that information from the electronic device is combined with MI-based feedback administered by a behavioral therapist. Feedback with EM devices has enhanced adherence to medication use in adult HIV, [8] adult hypertension [9] and pediatric asthma [10-12].

There have been no studies to date of adolescents or children with MS using EM with MIbased feedback as an interventional tool despite the critical benefit to be gained by maximizing adherence to DMT to prevent disability progression. Here, we report the results of a randomized controlled trial of EM plus MI feedback to enhance medication adherence in pediatric MS. 


\section{Methods}

This study was approved by the institutional review boards of all participating centers (Table 1). We recruited subjects from nine pediatric MS clinics from North America from October 2013 to January 2016 (Fig. 1). All patients were screened by local study personnel for eligibility and enrolled in the study if eligible and in agreement. Eligibility criteria included age 10-18, MS diagnosis using revised McDonald diagnostic criteria and International Pediatric MS Study Group criteria, $[13,14]$ and exposure to MS DMT for $\ 6$ months. Exclusion criteria were (1) use of IV DMT (e.g., natalizumab) or (2) being non-English speaking as our intervention was only available in English. Parent or guardian completed questionnaires complementary to the patient self-reports. Participants were randomized into either the behavioral feedback or attention control intervention. The randomization was stratified by: (1) oral versus injectable DMT; (2) whether the DMT injection is administered by the child's parent/guardian. Randomization took place after the baseline questionnaire was completed. A random number list for each of the strata was created (i.e., four columns of randomly assigned group listings) and assigned people in the order listed by strata. The study schema is shown in Fig. 2.

\section{Interventions}

The behavioral feedback + electronic monitoring device (MEMS cap) intervention was implemented from the Hospital for Sick Children by a group of behavioral interventionists who received certified MI training. Subjects received a supplemental device which downloaded their adherence data from the MEMS cap for use by the behavioral interventionist during a telephone feedback session. The behavioral interventionist scheduled three monthly telephone calls with participants at 1, 2 and 3 months postenrollment and, during each call, used a standard MI script which focused on goals related to DMT adherence and problem-solving around barriers to adherence. Parents were not involved in phone calls.

MI fidelity-Fidelity of the MI interviews was analyzed following established methods by a MI-trained psychologist blinded to subject status (adherence status) and interviewer. Transcribed interviews were coded according to the Motivational Interviewing Integrity (MITI) Code two times on two separate occasions. Fidelity scores were calculated using the following formulas: Global Spirit Score = (Evocation + Collaboration + Autonomy $/$ Support)/3; Proportion Complex Reflection = Complex Reflection/ $($ Complex Reflection + Simple Reflection); Proportion Open-ended Questions = Open-ended questions/(Openended questions + closed-ended questions); Proportion MI Adherent = Proportion of MI adherent counts/(Counts of MI Adherence + Counts of MI non-adherence); Ratio of Reflections to Questions $=($ Simple Reflections + Complex Reflections $) /($ Open-ended Questions + Closed-Ended Questions). Global ratings are rated on a scale of 1-5. Behavior counts are indicative of the number of times the outcome was used in the middle $20 \mathrm{~min}$ of the interview. Means were computed for each component on the MITI Coding Sheet as well as the composite scores recommended by the MITI [15]. Fidelity cutoffs recommended by the MITI were the following for beginning competency and competency, respectively: 
Global Spirit Score (3.5, 4); Proportion Complex Reflection (0.4, 0.5), Proportion Open Questions $(0.5,0.7)$, Proportion MI adherent $(0.9,1.0)$, Reflection:Question ratio $(1,2)$.

The attention control intervention consisted of a video related to DMT in pediatric MS. Use of this as the attention control intervention follows work that points to small to negligible effect sizes of purely educational approaches (mean $d=0.16,95 \% \mathrm{CI}=0.10-0.22$ ) and technological interventions (mean $d=0.08,95 \% \mathrm{CI}=0.09-0.25$ ) on adherence $[4,16]$. The participants were asked to complete a satisfaction questionnaire after the video to ensure completion of the task. The video was sent to the participants three times at 1,2 and 3 months post-enrollment by email as a link to the SurveyGizmo Web site.

\section{Measures}

Primary outcome-Adherence was measured using five objective and self-report methods focusing on different time frames and behaviors (Table 2). Objective sources of information included: (A) pharmacy refill data provided by site coordinators for 12 months prior to study entry and for 6 months post-study entry and (B) the MEMS cap, an EM device (MEMS, AARDEX) that captures each time the patient discards a needle from their injection or opens their pill bottle. Adherence information from MEMS caps is downloaded and stored on a secured web-platform (medAmigo ${ }^{\mathrm{TM}}$ ). These data were used to compile drug-dosing history data and to calculate medication adherence during the course of the study for baseline to one month; months 1-3; and months 3-6. Self-reported adherence from patients and parents included: (A) the Morisky Adherence Measure, a widely used 8-item patient-/parentreported measure with documented reliability and validity [17]. The following scoring algorithm was used: $8=$ high adherence, $6-7=$ medium adherence, and $<6=$ low adherence. (B) The Multiple Sclerosis Treatment Adherence Questionnaire (MSTAQ), which assesses missed doses, side effects and barriers of taking DMTs, and behavioral coping strategies used (e.g., icing the injection site, taking pain medication) over the past four weeks [18]. We adapted the MSTAQ to include both oral and injectable medications. We used a standardized scoring algorithm, where higher scores reflected numbers of missed doses, side effects, barriers, or behavioral coping strategies. Subjects completed only the barriers items, and the parent completed all items. (C) Parental involvement in DMT administration was tracked with the proportion of time (labeled as 0-25-50-75-100\%) the parent reported (1) reminding the child to take her/his DMT; (2) being present when the child took her/his DMT; and (3) administering the child's DMT.

Adherence definition-Whereas for most analyses we kept adherence variables continuous, for those analyses where we sought to characterize a non-adherent subgroup, we used the widely accepted cutoff for characterizing non-adherence as missing $20 \%$ of doses, either from pharmacy refill or from parent-reported data [19]. Because each adherence variable addressed a different time frame and they did not factor-analyze into one score, we analyzed the variables separately.

Secondary outcomes: quality of life and psychosocial outcomes-Secondary outcomes focused on patient-reported outcomes reflecting quality of life (QOL) and psychosocial well-being. Questionnaires were completed at baseline, 3 and 6 months. $Q O L$ 
was measured by: (1) the 23-item Pediatric QOL Inventory (PedsQL) measure of physical, social, emotional, and school functioning (Child/Teen report). The PedsQL has documented reliability and validity [20]. (2) Cognitive Functioning was assessed using the informantreport version of the MS Neuropsychological Screening Assessment Questionnaire (MSNQ) [21]. This tool has documented high test-retest stability and predictive and construct validity. Informant reports correlate with cognitive dysfunction and are less biased by patient depression [22].

Psychosocial outcomes-(a) the MS Self-Efficacy Scale (MSSE) is a reliable and valid 18-item measure of confidence in one's ability to manage disease symptoms (MSSE

Function subscale); and reactions to disease-related limitations and the impact of the disease on life activities (MSSE Control subscale); [23] (b) Ryff Scales of Psychological Well-Being (autonomy, environmental mastery and self-acceptance subscales), [24] a reliable and valid measure of well-being that has been used successfully with adolescents [25].

Covariates included demographics and the Patient-Determined Disease Steps (PDDS) [26] a measure that correlates highly with the Expanded Disability Status Scale (EDSS) [27]. It characterizes disability level into one of nine steps $(0=$ normal, $1=$ mild disability, $2=$ moderate disability, 3 = gait disability, $4=$ early cane, $5=$ late cane, $6=$ bilateral support, 7 $=$ wheelchair or scooter, $8=$ bedridden). An informant-reported version of the tool was administered to parent/guardians (the Flesch-Kincaid Grade Level estimate for the PDDS is grade 8.3 , suggesting a level appropriate for participants $>13$ years).

\section{Statistical analysis}

Descriptive statistics on the above measures were used to summarize the sample. We examined correlations among the measures of adherence. $\mathrm{T}$ tests were used to compare outcomes and demographic characteristics across groups (Table 1). We began by using longitudinal random effects models. Models were computed separately for objective adherence variables as well as parent- and patient-report using each of the above measures as dependent variables. Independent variables were group (behavioral intervention vs. control), time (baseline, 3, 6 months) and the interaction of group and time. We focused on the interaction term to examine whether the intervention had a differential effect over time.

Effect size and sample size calculations-Our sample size was sufficient to yield a large effect size (0.8), $80 \%$ power, $a=0.05$ [28]. In order to evaluate whether the intervention yielded small to medium effect sizes, we characterized mean changes in terms of Cohen's effect sizes (small $=0.20-0.49$; medium $=0.5-0.79$; and large $=0.80$ or larger $)$ [28].

Post hoc analyses sought to test whether the intervention had an impact on patient selfmanagement and to examine differences between those participating the full trial $(n=52)$ in comparison with those who dropped out $(n=14)$ for possible bias. Self-management analyses began by using factor analysis to create a self-management score for patients and parents. Random effects models and effect size computations were then used to examine whether there were differences over time on the self-management scores. Selection bias analyses were performed by computing effect sizes comparing mean baseline results from 
participants/parents who provided baseline data only as compared to those provided data for two or all three time points.

All analyses were performed using Stata 14 [29].

\section{Results}

\section{Sample characteristics}

The demographic characteristics of the two randomization groups were not significantly different (Table 1). Two-thirds of the sample was on an injectable DMT and one-third on an oral DMT. The sample had a low level of disability at baseline. Table 3 shows the descriptive statistics over time for the patient- and parent-reported outcomes.

\section{MI fidelity metrics}

Overall, the behavioral interventionists (MI facilitators) used for this study were at or above competency level in Global Spirit Score (4.12 \pm 0.3$)$, Proportion Complex Reflection (0.57 $\pm 0.13)$, Proportion Open Questions $(0.57 \pm 11)$ and lower than beginning competency in Proportion MI Adherent $(0.87 \pm 0.16)$, and Reflection: Question ratio $(0.69 \pm 0.4)$.

\section{Estimates of adherence and inter-correlations}

Depending on the adherence measure used, $1-41 \%$ of the sample was non-adherent at baseline. Those who were poor adherers using MEMS cap data at baseline tended to drop out. Among those with good adherence, those with lower adherence scores had lower adherence scores at 3- and 6-month follow-up (Fisher's exact $p=0.003,<0.0001$, respectively). Table 4 shows a correlation matrix with the adherence measures used in this study included.

\section{Impact of the intervention over time}

Primary outcomes-Results of the random effects models revealed significant or trend group-by-time interactions on MEMS cap adherence ( 6 months, $p<0.10$ ), parent-reported reminding ( 6 months, $p<0.05$ ), and administering ( 3 months, $p<0.10$ ) the DMT (Table 5). Table 2 shows group means at each time point as well as effect sizes. The intervention group decreased their MEMS cap adherence, while increasing how often their parents reminded and administered their DMT. The intervention group exhibited lower MEMS cap adherence (both at 3 and at 6 months) but better pharmacy refill adherence (at 6 months) compared to the control group, and no difference on parent-reported proportion missed doses over time (Fig. 3). Parents of youth receiving the behavioral intervention reported increasing reminding (3-6 months, $d=0.59,0.70$ ), being present ( 6 months, $d=0.35$ ), and administering the DMT ( 3 and 6 months, $d=0.33,0.29$ ) over time, better Morisky adherence ( 3 months, $d=0.21)$, more MSTAQ Barriers $(d=0.45)$ and MSTAQ Side Effects $(d=0.44)$, and fewer MSTAQ Behavioral Coping Strategies ( 3 months, $d=-0.39,6$ months $d=-0.68)$, compared to the control group. While the behavioral intervention patients also reported better Morisky adherence ( 3 months, $d=0.21$ ), they reported fewer MSTAQ Barriers ( 3 months, $d=-0.58,6$ months, $d=-0.31$ ) over time compared to the control group (Table 2). 
Secondary outcomes-Results of the random effect models revealed significant or trend group-by-time interactions on patient-reported PedsQL physical functioning (6 months, $p$ $<0.10$ ) and MSSE Control (3 months, $p<0.05$; Table 5). An examination of mean changes shows that intervention patients reported better MSSE Functioning $(d=0.64)$ and MSSE control $(d=0.43)$ compared to the control group (Table 2$)$. The effect size comparisons revealed that intervention patients had worse parent-reported MSNQ cognitive and PedsQL school functioning over time (6 months, $d=-0.22$, and 3 months, $d=-0.35$, respectively), but improved PedsQL Physical Functioning ( 3 months, $d=0.36$ and 6 months, $d=0.21$ ) compared to the control group. Intervention patients reported better MSSE Function and control ( 3 months, $d=0.55,0.54$, and 6 months, $d=0.48,0.21$ respectively), and better PedsQL Physical ( 3 months, $d=0.23$ and 6 months, $d=0.45$ ), Emotional ( 3 months, $d=$ 0.25 ) and Social ( 3 months, $d=0.25$ ) Functioning. Conversely, they reported worse wellbeing outcomes, i.e., lower Ryff Self-Acceptance (6 months, $d=-0.53$ ) and Ryff Environmental Mastery ( 3 months, $d=-0.42$ and 6 months, $d=-0.42$ ), compared to control patients (Table 2).

Changes in self-management-Rotated factor analyses created two parent- and two patient-reported self-management scores. The first parent-reported self-management factor score-Behavioral Involvement—summarized the Present, Administer, and MSTAQ Behavioral Coping scores, and the second factor score-Cognitive Involvementsummarized the Remind and MSNQ Cognitive Function scores (eigenvalues $=2.18$ and 1.24). The first patient-reported self-management factor score-Self-Efficacy-summarized the MSSE Function and control scores, and the second factor score-Well-Beingsummarized the Ryff Autonomy, Self-Acceptance, and Environmental Mastery scores (eigenvalues $=1.89$ and 1.52). Results of the random effect models revealed significant or trend group-by-time interactions on Cognitive Involvement (6 months, $p<0.05$ ) and Selfefficacy ( 3 months, $p<0.10$ ). Effect size comparisons revealed that the intervention group had more Cognitive Involvement at 6 months, better Self-efficacy at 3 and 6 months, but worse Well-Being at 3 and 6 months, compared to the control group (Table 2).

Selection biases-While an intention-to-treat analysis is ideal, it was not possible to implement such in the present study because 14 (21\%) of the 66 randomized participants provided only baseline data. A next best alternative is to examine potential selection biases in the study sample. Table 6 shows results of effect size comparisons of the sample lost to follow-up with the analytic sample. It revealed that patients who opted out of the study after randomization were younger and had parents with less-than-college education. They were more likely to speak English as a primary language and less likely to have an Individualized Education Program. On the primary adherence outcomes, they had lower adherence on "pharmacy refills" and on "proportion missed doses," higher utilization of behavioral coping, worse side effects, worse barriers (parent and patient), and worse patient-reported Morisky adherence scores. Those lost to follow-up also had worse parent-reported PedsQL Physical, Emotional, Social, and School Functioning. They had worse patient-reported MSSE Control, Ryff Autonomy, Self-Acceptance and Environmental Mastery, and worse PedsQL Physical, Emotional, and School Functioning (Table 6). 


\section{Discussion}

While youth participating in our trial started with and maintained high levels of medication adherence using most adherence measures, several interesting and potentially contradictory findings emerged. We found improvements which were sustained at 3 and 6 months in selfefficacy (MSSE function and control) and patient- and parent-reported physical function, but found sustained decreases in patient-reported environmental mastery in those receiving the intervention versus controls. Small deterioration in parent-reported school function and small improvements in patient-reported emotional and social function were seen at 3 months but not sustained at 6 months.

Adherence rates in most measures showed small effect size changes. We found a modest effect on rates of pharmacy refills and parent-reported adherence (Morisky) at three months, which was not sustained at six months, in the intervention group compared to the control group. However, surprisingly there was a marked drop in use of the EM device in the last three months of the study in the intervention group but not the control group. Reasons for the discrepancy in MEMS cap data versus self-report and refill data are unknown, but may include less attention to day-today study procedures following the completion of the behavioral intervention cycle of interviews, or a true decrease in medication adherence due to the lack of the behavioral interventionist reminding the patient to use the study materials. Remarkably, we saw increased parental reminders at the 6-month mark in comparison with the 3-month mark, with concomitant increasing drop-off in MEMS cap use in subjects. The directionality of this association is unknown: Did parents remind more frequently due to noticed drop-off in medication use OR did children decrease their medication use as parents increased their reminders? Differences between the behavior of the intervention versus control groups suggest the need for further, more granular analysis about what may have led to the behavioral change in the intervention group. This question will be addressed in a phenomenological assessment of interview data from this cohort.

Development of self-management skills constitutes one of the key elements to successful transition to adulthood and is especially important in youth with chronic illness [30]. While the primary goal of the trial was to change adherence, methods used in our intervention were aimed at providing tools for increasing self-management. We found some important secondary effects of the intervention including increased skills that may improve selfmanagement, as reflected in increased self-efficacy scores. Concomitantly and perhaps reflecting increased parental involvement in adherence, parents of the intervention group reported decreases in cognitive and school functioning over time, and the children reported decreased self-acceptance and environmental mastery. This phenomenon was not seen in the control group.

Furthermore, parent reports from intervention participants described more barriers, side effects and fewer behavioral coping strategies. It is possible that even if parents were not involved in behavioral feedback calls, use of MI led to greater parental involvement and changes in awareness of cognitive challenges that the youth were facing. Whether this increase in parental involvement was beneficial or detrimental to self-management remains to be seen, but decreases in MEMS cap use by intervention participants suggest the need to 
explore the effect of increasing parental involvement on medication adherence. Design of future interventions involving adolescents with MS should consider this striking observation.

Importantly, we found inconsistency between parents and patients on several outcomes, including barriers to adherence, PedsQL Emotional, Social, and School Function, which suggests that parents and children interpret their experiences differently and/or attend to different factors when evaluating the same variables/items. This phenomenon has been widely reported [31,32] and emphasizes the need to seek both parent and child perspectives in research on evaluative constructs such as quality of life.

One of the most striking findings from our study was that youth who may have benefited most from the intervention dropped out from the study after consent. Youth in the group lost to follow-up represented $21 \%$ of the consented population. They were more likely to be nonadherent, had lower parental education, lower parent-reported and self-reported quality of life scores, and lower self-reported self-efficacy (MSSE control) and autonomy, selfacceptance, and environmental mastery scores (Ryff). The study was explicitly set up to address concerns of attrition and participation by offering a flexible, telephone-based intervention, perhaps mitigating a higher attrition rate. Nonetheless, based on these findings, it is clear that barriers unique to documented characteristics of those lost to follow-up must be addressed in future studies.

This study has limitations. The relatively few statistically significant differences comparing intervention and control groups are likely due to low power to detect the medium to small effect sizes generally found in behavioral intervention studies [33, 34]. In quality-of-life studies, a moderate effect size is generally considered to be a clinically relevant [35]. Our analysis focus on characterizing effect sizes yields valuable information on the impact of the intervention on change, some of which are clinically important and others of which are small effect sizes that are relevant nonetheless [35].

In addition, blinded fidelity assessment of the MI interviews suggested greater adherence to MI principles in some domains of fidelity than others: The intervention therefore could have had a greater effect if there had been a higher level of overall fidelity to these principles.

Duration of follow-up was limited to 6 months. Finally, the high rates of baseline adherence in our cohort not only restricted our ability to improve adherence levels, but also make regression to the mean a possible explanation for any putative worsening in adherence outcomes.

In summary, in this cohort of youth with MS, increased pharmacy refills, increases in selfreported adherence, increases in quality of life and self-efficacy but decreases in well-being and use of an EM device were documented after the use of an intervention combining an EM device and MI-based feedback. This may have long-term implications for youth with MS, as these factors play strongly into future independence and disease management. Strategies for engaging youth and maintaining their engagement are important and necessary in future trials. 


\section{Acknowledgments}

We are grateful for the involvement of the youth with MS and their parents, as well as to all the investigators and their institutions involved, without whom this study would not have been possible.

Funding This work was funded by the National Multiple Sclerosis Society (HC 0148).

\section{Abbreviations}

$\begin{array}{ll}\text { DMT } & \text { Disease-modifying therapy } \\ \text { EM } & \text { Electronic monitoring } \\ \text { MI } & \text { Motivational interviewing } \\ \text { MS } & \text { Multiple sclerosis } \\ \text { MSSE } & \text { MS Self-Efficacy Scale } \\ \text { MSTAQ } & \text { Multiple Sclerosis Treatment Adherence Questionnaire } \\ \text { PedsQL } & \text { Pediatric Quality of Life Inventory } \\ \text { SD } & \text { Standard deviation }\end{array}$

\section{References}

1. Yeh EA, Waubant E, Krupp LB, Ness J, Chitnis T, Kuntz N, et al. Multiple sclerosis therapies in pediatric patients with refractory multiple sclerosis. Archives of Neurology. 2011; 68(4):437-444. DOI: 10.1001/archneurol.2010.325 [PubMed: 21149803]

2. Thannhauser JE, Mah JK, Metz LM. Adherence of adolescents to multiple sclerosis diseasemodifying therapy. Pediatric Neurology. 2009; 41(2):119-123. DOI: 10.1016/j.pediatrneurol. 2009.03.004 [PubMed: 19589460]

3. Giovannoni G, Southam E, Waubant E. Systematic review of disease-modifying therapies to assess unmet needs in multiple sclerosis: Tolerability and adherence. Multiple Sclerosis Journal. 2012; 18(7):932-946. [PubMed: 22249762]

4. Kahana S, Drotar D, Frazier T. Meta-analysis of psychological interventions to promote adherence to treatment in pediatric chronic health conditions. Journal of Pediatric Psychology. 2008; 33(6): 590-611. DOI: 10.1093/jpepsy/jsm128 [PubMed: 18192300]

5. Kahana S, Drotar D, Frazier T. Meta-analysis of psychological interventions to promote adherence to treatment in pediatric chronic health conditions. Journal of Pediatric Psychology. 2008; 33(6): 590-611. [PubMed: 18192300]

6. Graves MM, Roberts MC, Rapoff M, Boyer A. The efficacy of adherence interventions for chronically ill children: A meta-analytic review. Journal of Pediatric Psychology. 2010; 35(4):368382. [PubMed: 19710248]

7. Britt E, Hudson SM, Blampied NM. Motivational interviewing in health settings: A review. Patient Education and Counseling. 2004; 53(2):147-155. DOI: 10.1016/S0738-3991(03)00141-1 [PubMed: 15140454]

8. Sabin LL, DeSilva MB, Hamer DH, Xu K, Zhang J, Li T, et al. Using electronic drug monitor feedback to improve adherence to antiretroviral therapy among HIV-positive patients in China. AIDS and Behavior. 2010; 14(3):580-589. DOI: 10.1007/s10461-009-9615-1 [PubMed: 19771504]

9. Ruppar TM. Randomized pilot study of a behavioral feedback intervention to improve medication adherence in older adults with hypertension. Journal of Cardiovascular Nursing. 2010; 25(6):470 479. DOI: 10.1097/JCN.0b013e3181d5f9c5 [PubMed: 20856132] 
10. Bartlett SJ, Lukk P, Butz A, Lampros-Klein F, Rand CS. Enhancing medication adherence among inner-city children with asthma: Results from pilot studies. Journal of Asthma. 2002; 39(1):47-54. [PubMed: 11883739]

11. Burgess SW, Sly PD, Devadason SG. Providing feedback on adherence increases use of preventive medication by asthmatic children. Journal of Asthma. 2010; 47(2):198-201. DOI: 10.3109/02770900903483840 [PubMed: 20170329]

12. Herzer M, Ramey C, Rohan J, Cortina S. Incorporating electronic monitoring feedback into clinical care: A novel and promising adherence promotion approach. Clinical child psychology and psychiatry. 2011; doi: 10.1177/1359104511421103

13. Krupp LB, Banwell B, Tenembaum S. Consensus definitions proposed for pediatric multiple sclerosis and related disorders. Neurology. 2007; 68(16 Suppl 2):S7-12. DOI: 10.1212/01.wnl. 0000259422.44235.a8 [PubMed: 17438241]

14. Polman CH, Reingold SC, Banwell B, Clanet M, Cohen JA, Filippi M, et al. Diagnostic criteria for multiple sclerosis: 2010 revisions to the McDonald criteria. Annals of Neurology. 2011; 69(2): 292-302. DOI: 10.1002/ana.22366 [PubMed: 21387374]

15. Moyers TB, Manuel JK, Ernst D. Motivational Interviewing Treatment Integrity Coding Manual 4.1. 2014.

16. Graves MM, Roberts MC, Rapoff M, Boyer A. The efficacy of adherence interventions for chronically ill children: A meta-analytic review. Journal of Pediatric Psychology. 2010; 35(4):368382. DOI: 10.1093/jpepsy/jsp072 [PubMed: 19710248]

17. Morisky DE, Ang A, Krousel-Wood M, Ward HJ. Predictive validity of a medication adherence measure in an outpatient setting. The Journal of Clinical Hypertension. 2008; 10(5):348-354. [PubMed: 18453793]

18. Wicks P, Massagli M, Kulkarni A, Dastani H. Use of an online community to develop patientreported outcome instruments: the Multiple Sclerosis Treatment Adherence Questionnaire (MSTAQ). Journal of medical Internet research. 2011; 13(1):e12. [PubMed: 21266318]

19. McKay KA, Tremlett H, Patten SB, Fisk JD, Evans C, Fiest K, et al. Determinants of nonadherence to disease-modifying therapies in multiple sclerosis: A cross-Canada prospective study. Multiple Sclerosis Journal. 2016; 23(4):588-596. DOI: 10.1177/1352458516657440 [PubMed: 27357507]

20. Varni JW, Seid M, Kurtin PS. PedsQL ${ }^{\mathrm{TM}} 4.0$ : Reliability and validity of the Pediatric Quality of Life Inventory ${ }^{\mathrm{TM}}$ Version 4.0 Generic Core Scales in healthy and patient populations. Medical Care. 2001; 39(8):800-812. [PubMed: 11468499]

21. Benedict RH, Cox D, Thompson LL, Foley F, Weinstock-Guttman B, Munschauer F. Reliable screening for neuropsychological impairment in multiple sclerosis. Multiple Sclerosis Journal. 2004; 10(6):675-678. [PubMed: 15584493]

22. O’Brien A, Gaudino-Goering E, Shawaryn M, Komaroff E, Moore NB, DeLuca J. Relationship of the Multiple Sclerosis Neuropsychological Questionnaire (MSNQ) to functional, emotional, and neuropsychological outcomes. Archives of Clinical Neuropsychology. 2007; 22(8):933-948. [PubMed: 17851031]

23. Schwartz CE, Coulthard-Morris L, Zeng Q, Retzlaff P. Measuring self-efficacy in people with multiple sclerosis: a validation study. Archives of Physical Medicine and Rehabilitation. 1996; 77(4):394-398. DOI: 10.1016/S0003-9993(96)90091-X [PubMed: 8607766]

24. Ryff CD. Happiness is everything, or is it? explorations on the meaning of psychological wellbeing. Journal of Personality and Social Psychology. 1989; 57:1069-1081.

25. Schwartz CE, Keyl P, Bode R, Marcum J. Helping others shows differential benefits on health and well-being for male and female teens. Journal of Happiness Studies. 2009; 10(4):431-448.

26. Hohol MJ, Orav EJ, Weiner HL. Disease steps in multiple sclerosis: A simple approach to evaluate disease progression. Neurology. 1995; 45:251-255. [PubMed: 7854521]

27. Schwartz CE, Vollmer T, Lee H. Reliability and validity of two self-report measures of impairment and disability for MS. North American Research Consortium on Multiple Sclerosis Outcomes Study Group. Neurology. 1999; 52(1):63-70. [PubMed: 9921850]

28. Cohen J. A power primer. Psychological Bulletin. 1992; 112:155-159. [PubMed: 19565683]

29. StataCorp. Stata statistical software: release 14. College Station: StataCorp LP; 2016. 
30. Nazareth M, Richards J, Javalkar K, Haberman C, Zhong Y, Rak E, et al. Relating health locus of control to health care use, adherence, and transition readiness among youths with chronic conditions, North Carolina, 2015. Preventing chronic disease. 2016; 13:E93.doi: 10.5888/ pcd13.160046 [PubMed: 27442993]

31. Klassen AF, Miller A, Fine S. Agreement between parent and child report of quality of life in children with attention-deficit/hyperactivity disorder. Child: Care, Health and Development. 2006; 32(4):397-406. DOI: 10.1111/j.1365-2214.2006.00609.x

32. Jozefiak T, Larsson B, Wichstrom L, Mattejat F, Ravens-Sieberer U. Quality of Life as reported by school children and their parents: A cross-sectional survey. Health and Quality of Life Outcomes. 2008; 6:34.doi: 10.1186/1477-7525-6-34 [PubMed: 18489777]

33. Meyer TJ, Mark MM. Effects of psychosocial interventions with adult cancer patients: a metaanalysis of randomized experiments. Health Psychology. 1995; 14(2):101-108. [PubMed: 7789344]

34. Grossman P, Niemann L, Schmidt S, Walach H. Mindfulness-based stress reduction and health benefits A meta-analysis. Journal of psychosomatic research. 2004; 57(1):35-43. DOI: 10.1016/ S0022-3999(03)00573-7 [PubMed: 15256293]

35. Norman GR, Sloan JA, Wyrwich KW. Interpretation of changes in health-related quality of life: The remarkable universality of half a standard deviation. Medical Care. 2003; 41(5):582-592. DOI: 10.1097/01.MLR.0000062554.74615.4C [PubMed: 12719681]

\section{Pediatric MS Adherence Study Group}

Gregory Aaen, Gulay Alper, Brenda L. Banwell, Charlene Belsole, Tara Berenbaum, Petra Breiner, Susana Camposano, Hardeep Chohan, Carolynn Darrell, Sarah Dowdy, Kim Edwards, Mark Gorman, Jennifer Graves, La June Grayson, Stephanie A. Grover, Tiffany Haig, Sabrina Hamer, Janace Hart, Kawonas Jenkins, Amy Lavery, Geraldine Liu, Timothy Lotze, Jean K. Mah, Rory Mahabir, Soe Mar, Lauren Mednick, Elva R. Mendoza, Manikum Moodley, Jayne Ness, Austin Noguera, Maya Obadia, Marvin Petty, Sarah Planchon Pope, Daniela Pohl, Mariam Pontifes, Victoria E. Powell, Elizabeth Quon, Mary Rensel, Jennifer Resto, Ian Rossman, Melissa Rundquist, Karla Sanchez, Teri Schreiner, Carolyn E. Schwartz, Ruth Slater, Maleka Smith, Jaime Sorum, Alexander Stein, Marija Stosic, JanMendelt Tillema, Sunita Venkateswaran, Jennifer Vincent, Amy Waldman, Emmanuelle Waubant and E. Ann Yeh. 


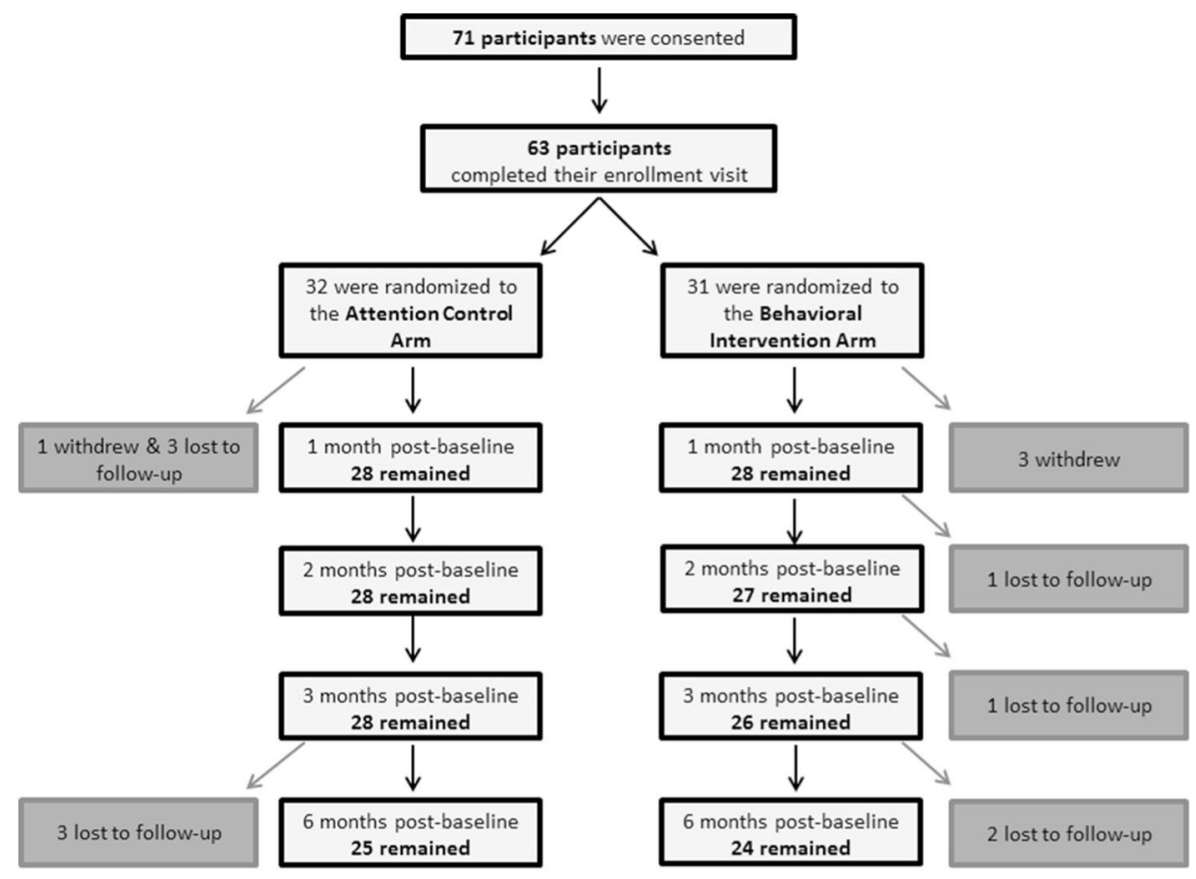

Fig. 1.

Recruitment flow chart 

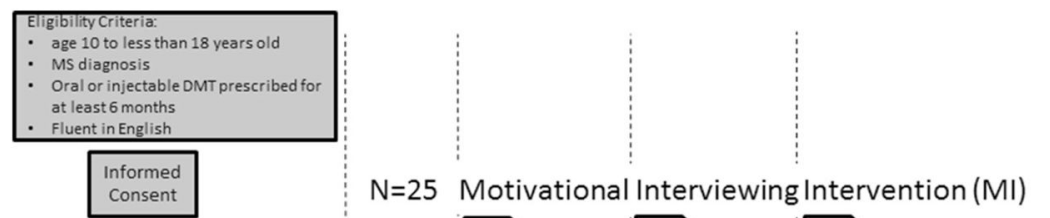

Randomization

Stratified by:

- Oral versus injectable DMT

- Parent administered DMT or not

$\mathrm{N}=25$ Motivational Interviewing Intervention (MI)
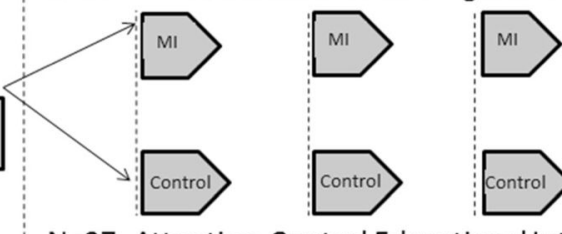

$\mathrm{N}=27$ Attention-Control Educational Intervention (Control)

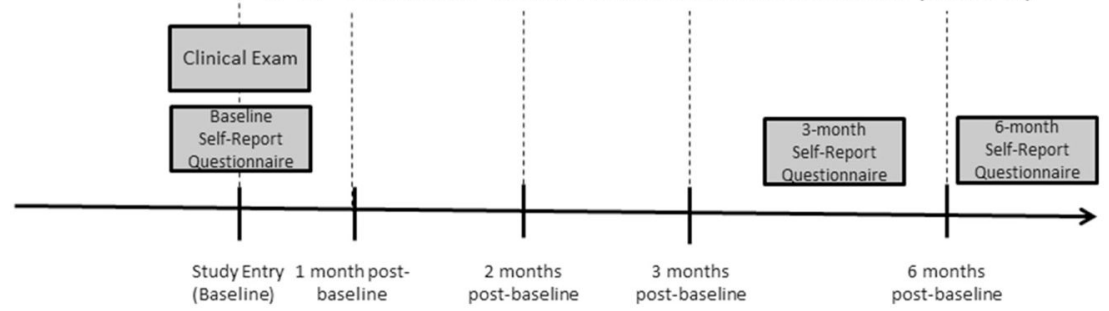

Fig. 2.

Study schema 


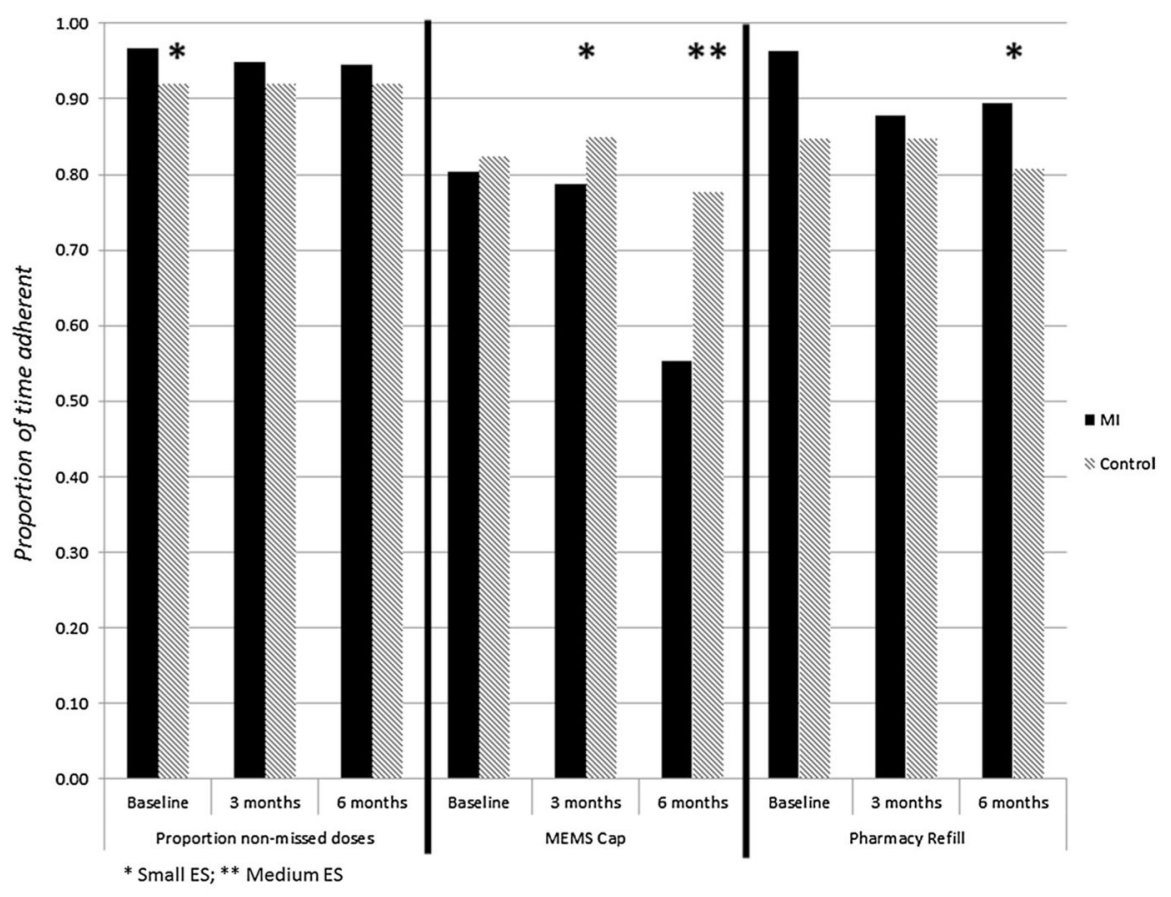

Fig. 3.

Bar chart showing adherence results 
Table 1

Sample demographics by randomization group

\begin{tabular}{|c|c|c|c|c|}
\hline Variable & $\begin{array}{l}\text { Whole sample }(n= \\
\text { 52) }\end{array}$ & Control $(n=27)$ & $\begin{array}{l}\text { Intervention }(n= \\
\text { 25) }\end{array}$ & $p$ value \\
\hline Mean age (sd) & $16.03(2.20)$ & $15.76(2.52)$ & $16.32(1.81)$ & 0.37 \\
\hline Mean age at diagnosis (sd) & $13.62(2.27)$ & $13.18(2.28)$ & $14.11(2.21)$ & 0.14 \\
\hline Mean age at menarche (sd) & $11.55(1.21)$ & $11.50(1.15)$ & $11.60(1.30)$ & 0.82 \\
\hline $\begin{array}{l}\text { How often did you remind your child to take his/her } \\
\text { medications? (proportion of time, expressed in \%) (mean, sd) }\end{array}$ & $44.61(38.83)$ & $51.85(40.98)$ & $36.46(35.34)$ & 0.16 \\
\hline $\begin{array}{l}\text { How often were you present during the administration of your } \\
\text { child's medication? (proportion of time, expressed in \%) } \\
\text { (mean, sd) }\end{array}$ & $65.20(37.11)$ & $66.67(34.67)$ & $63.54(40.36)$ & 0.77 \\
\hline $\begin{array}{l}\text { How often did you administer the medication to your child? } \\
\text { (proportion of time, expressed in \%) (mean, sd) }\end{array}$ & $36.27(41.33)$ & $37.96(41.82)$ & $34.38(41.58)$ & 0.76 \\
\hline Mean PDDS (sd) & $0.47(0.84)$ & $0.60(0.91)$ & $0.33(0.76)$ & 0.27 \\
\hline Mean informant-reported MSNQ (sd) & $17.90(13.42)$ & $17.81(13.84)$ & $18.0(13.23)$ & 0.96 \\
\hline Mean EDSS (sd) & $1.23(1.01)$ & $1.09(0.94)$ & $1.38(1.07)$ & 0.31 \\
\hline \multicolumn{5}{|l|}{ Gender ( $\%$ female) } \\
\hline Female & 65.38 & 59.26 & 72 & 0.34 \\
\hline \multicolumn{5}{|l|}{ Race (\% White) } \\
\hline White & 44.23 & 40.74 & 48 & 0.60 \\
\hline \multicolumn{5}{|l|}{ Mother education (\%) } \\
\hline Less than college & 50 & 55.56 & 44 & 0.40 \\
\hline College degree or more & 46.15 & 40.74 & 52 & \\
\hline Missing & 3.85 & 3.7 & 4 & \\
\hline \multicolumn{5}{|l|}{ Father education (\%) } \\
\hline Less than college & 61.54 & 74.07 & 48 & 0.08 \\
\hline College degree or more & 36.54 & 25.93 & 48 & \\
\hline Missing & 1.92 & 0 & 4 & \\
\hline \multicolumn{5}{|l|}{ Mode of administration (\%) } \\
\hline Paper and pencil & 21.15 & 25.93 & 16 & 0.34 \\
\hline Computer & 76.92 & 70.37 & 84 & \\
\hline Missing & 1.92 & 3.7 & 0 & \\
\hline \multicolumn{5}{|l|}{ Tobacco use (\%) } \\
\hline No & 90.38 & 92.59 & 88 & 0.80 \\
\hline Yes, occasionally & 3.85 & 3.7 & 4 & \\
\hline Prefer not to answer & 5.77 & 3.7 & 8 & \\
\hline \multicolumn{5}{|l|}{ Primary language (\%) } \\
\hline English & 82.69 & 85.19 & 80 & 0.55 \\
\hline Spanish & 1.92 & 3.7 & 0 & \\
\hline Other & 13.46 & 11.11 & 16 & \\
\hline Missing & 1.92 & 0 & 4 & \\
\hline \multicolumn{5}{|l|}{ Individualized educational program (\%) } \\
\hline No & 59.62 & 55.56 & 64 & 0.31 \\
\hline
\end{tabular}




\begin{tabular}{|c|c|c|c|c|}
\hline Variable & $\begin{array}{l}\text { Whole sample }(n= \\
\text { 52) }\end{array}$ & Control $(n=27)$ & $\begin{array}{l}\text { Intervention }(n= \\
\text { 25) }\end{array}$ & $p$ value \\
\hline Yes & 36.54 & 44.44 & 28 & \\
\hline Missing & 3.85 & 0 & 8 & \\
\hline \multicolumn{5}{|l|}{ Medication type (\%) } \\
\hline Injectable & 73 & 78 & 68 & 0.43 \\
\hline $\begin{array}{l}\text { Avonex or Avonex pre-filled syringe (Interferon Beta1a- } \\
\text { intramuscular) }\end{array}$ & 18.42 & 24 & 12 & \\
\hline Copaxone (Glatiramer acetate) & 55.26 & 52 & 59 & \\
\hline PLEGRIDY (peginterferon beta-1a) & 7.89 & 0 & 18 & \\
\hline Rebif (Interferon Betalb -subcutaneous) & 18.42 & 24 & 12 & \\
\hline Oral & 27 & 22 & 32 & \\
\hline Gilenya (fingolimod) & 15 & 33 & 71 & \\
\hline Tecfidera (BG-12 or dimethyl fumarate) & 69 & 67 & 29 & \\
\hline Terifluonomide & 15 & 0 & 0 & \\
\hline \multicolumn{5}{|l|}{ Site $(\%)$} \\
\hline Hospital for Sick Children, Toronto & 40.38 & 37.04 & 44 & 0.41 \\
\hline Childrens Hospital of Philadelphia & 5.77 & 3.7 & 8 & \\
\hline Childrens Hospital of Pittsburgh & 3.85 & 7.41 & 0 & \\
\hline Boston Childrens Hospital & 11.54 & 7.41 & 16 & \\
\hline University of Alabama at Birmingham & 11.54 & 18.52 & 4 & \\
\hline University of Colorado Denver & 5.77 & 3.7 & 8 & \\
\hline University of California at San Francisco & 5.77 & 7.41 & 4 & \\
\hline Texas Childrens Hospital, Baylor College of Medicine & 11.54 & 14.81 & 8 & \\
\hline Alberta Childrens Hospital & 3.85 & 0 & 8 & \\
\hline
\end{tabular}

$s d$ standard deviation 


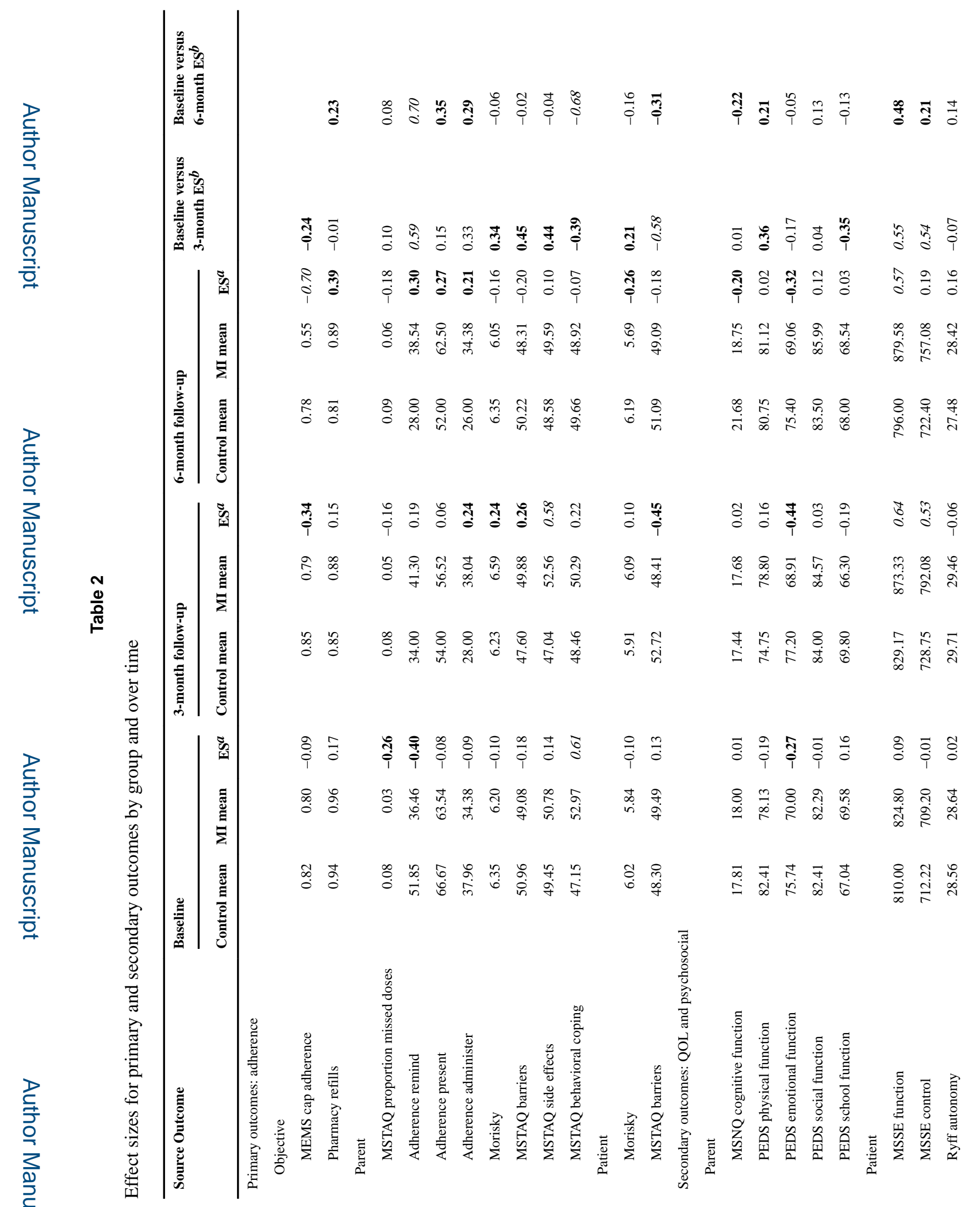

Qual Life Res. Author manuscript; available in PMC 2018 September 21. 
Yeh et al.

Page 20

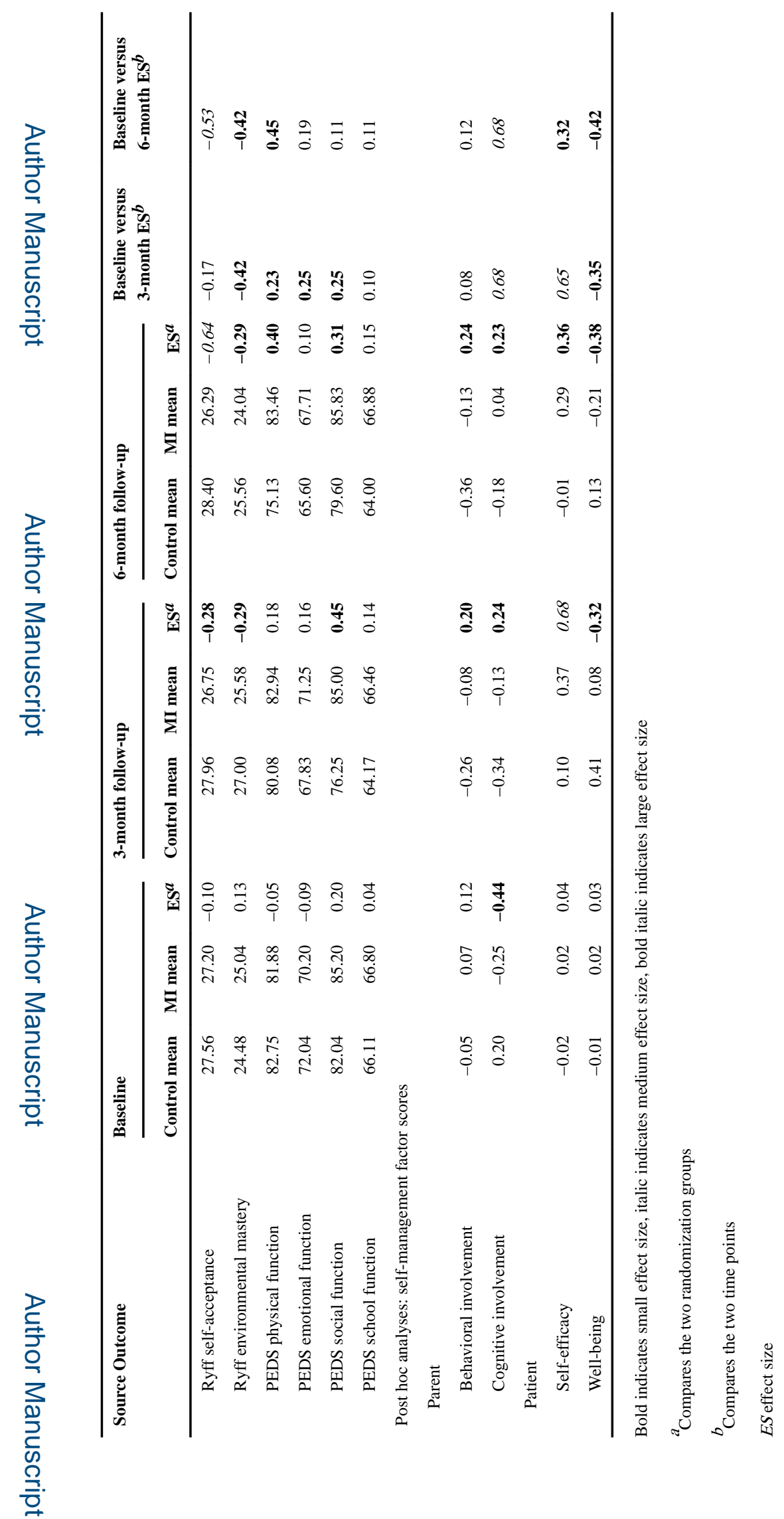

Qual Life Res. Author manuscript; available in PMC 2018 September 21. 


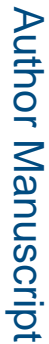

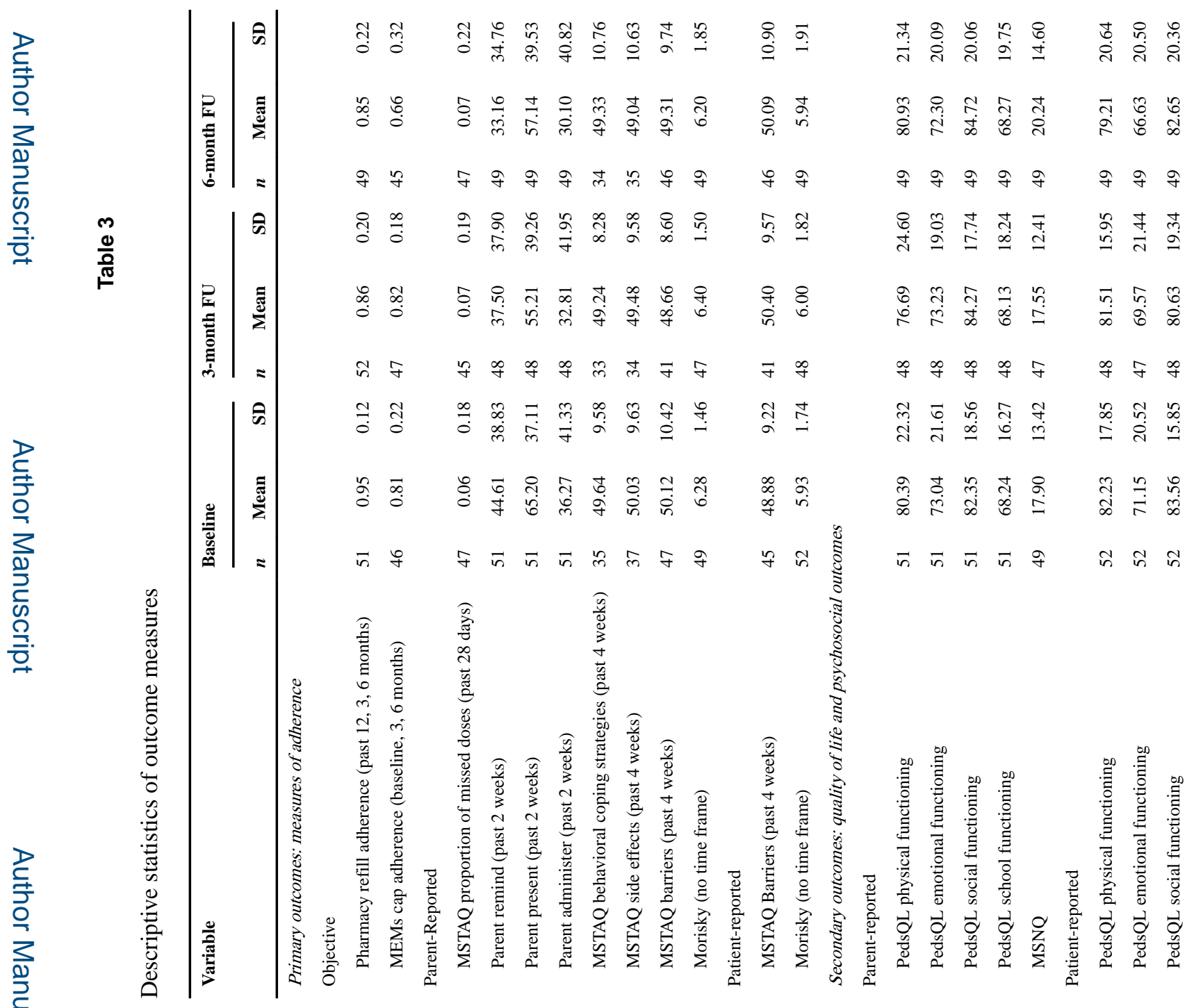

Qual Life Res. Author manuscript; available in PMC 2018 September 21. 


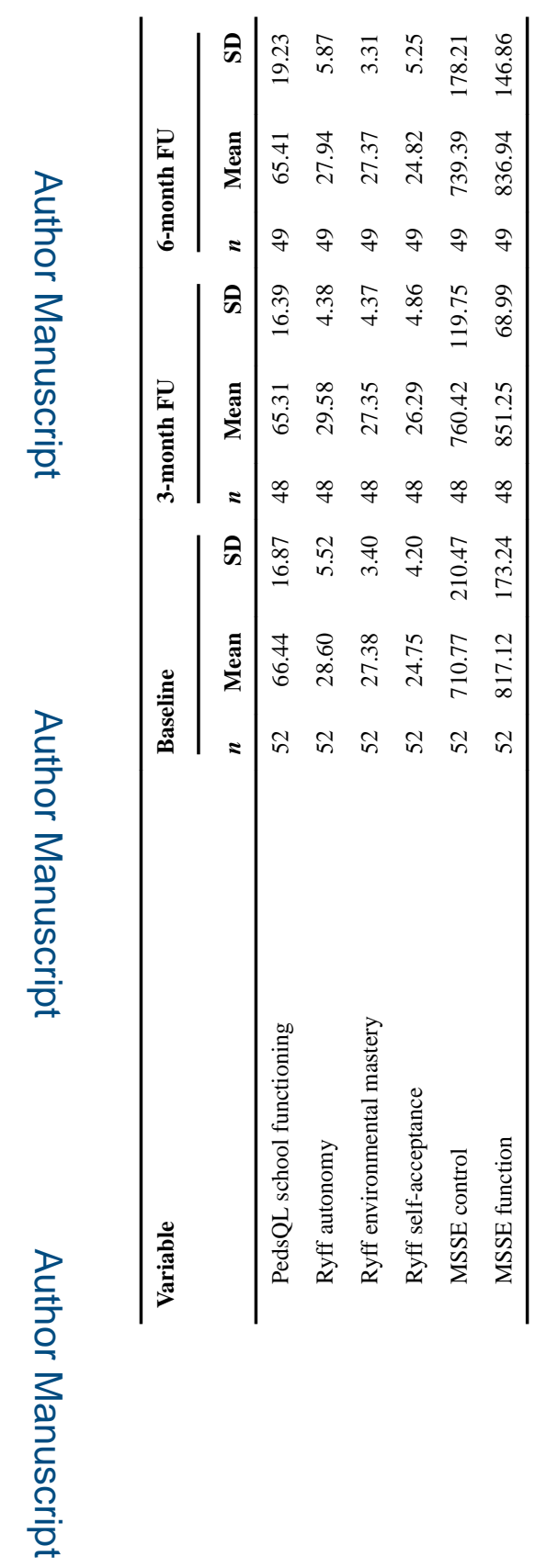

로을

Qual Life Res. Author manuscript; available in PMC 2018 September 21. 


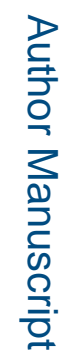

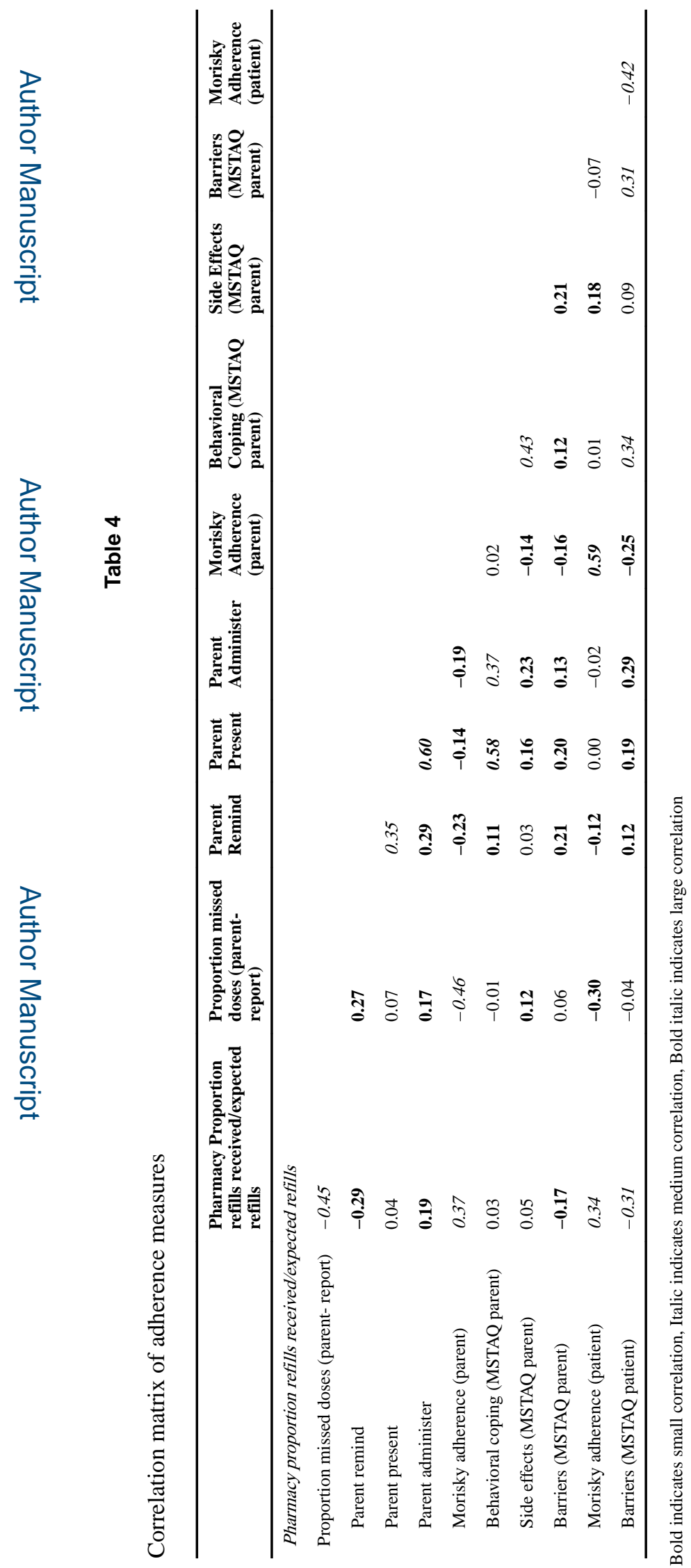




\section{Table 5}

Results of random effect models

\begin{tabular}{|c|c|c|}
\hline Outcome & Months & Group $\times$ Time Interaction \\
\hline \multicolumn{3}{|l|}{ Primary outcomes: measures of adherence } \\
\hline \multicolumn{3}{|l|}{ Objective } \\
\hline \multirow[t]{2}{*}{ Pharmacy refills } & 3 & -0.02 \\
\hline & 6 & 0.07 \\
\hline \multirow[t]{2}{*}{ MEMs cap } & 3 & 0.02 \\
\hline & 6 & $-0.19^{* * *}$ \\
\hline \multicolumn{3}{|l|}{ Parent-reported } \\
\hline \multirow[t]{2}{*}{ MSTAQ proportion missed doses } & 3 & 0.01 \\
\hline & 6 & 0.01 \\
\hline \multirow[t]{2}{*}{ Parent remind } & 3 & 17.65 \\
\hline & 6 & $26.01^{*}$ \\
\hline \multirow[t]{2}{*}{ Parent present } & 3 & 1.94 \\
\hline & 6 & 12.63 \\
\hline \multirow[t]{2}{*}{ Parent administer } & 3 & $11.69^{* *}$ \\
\hline & 6 & 12.85 \\
\hline \multirow[t]{2}{*}{ MSTAQ behavioral coping strategies } & 3 & -3.69 \\
\hline & 6 & -4.66 \\
\hline \multirow[t]{2}{*}{ MSTAQ side effects } & 3 & 0.64 \\
\hline & 6 & -1.35 \\
\hline \multirow[t]{2}{*}{ MSTAQ barriers } & 3 & 0.99 \\
\hline & 6 & -0.03 \\
\hline \multirow[t]{2}{*}{ Morisky } & 3 & 0.34 \\
\hline & 6 & -0.26 \\
\hline \multicolumn{3}{|l|}{ Patient-reported } \\
\hline \multirow[t]{2}{*}{ MSTAQ barrier } & 3 & -2.90 \\
\hline & 6 & -3.56 \\
\hline \multirow[t]{2}{*}{ Morisky } & 3 & 0.20 \\
\hline & 6 & -0.35 \\
\hline \multicolumn{3}{|c|}{ Secondary outcomes: quality of life and psychosocial outcomes } \\
\hline \multicolumn{3}{|l|}{ Parent-reported } \\
\hline \multirow[t]{2}{*}{ PedsQL physical function } & 3 & 1.63 \\
\hline & 6 & 3.93 \\
\hline \multirow[t]{2}{*}{ PedsQL emotional function } & 3 & -0.92 \\
\hline & 6 & -0.44 \\
\hline \multirow[t]{2}{*}{ PedsQL social function } & 3 & 1.03 \\
\hline & 6 & 2.19 \\
\hline \multirow[t]{2}{*}{ PedsQL school function } & 3 & -4.51 \\
\hline & 6 & -2.90 \\
\hline MSNQ & 3 & -1.85 \\
\hline
\end{tabular}




\begin{tabular}{|c|c|c|}
\hline Outcome & Months & Group $\times$ Time Interaction \\
\hline & 6 & -3.27 \\
\hline \multicolumn{3}{|l|}{ Patient-reported } \\
\hline \multirow[t]{2}{*}{ PedsQL physical function } & 3 & 4.93 \\
\hline & 6 & $9.13^{\text {** }}$ \\
\hline \multirow[t]{2}{*}{ PedsQL emotional function } & 3 & 4.21 \\
\hline & 6 & 3.11 \\
\hline \multirow[t]{2}{*}{ PedsQL social function } & 3 & 1.11 \\
\hline & 6 & 1.42 \\
\hline PedsQL school function & Model $d$ & not converge \\
\hline \multirow[t]{2}{*}{ MSSE function } & 3 & 56.21 \\
\hline & 6 & 68.53 \\
\hline \multirow[t]{2}{*}{ MSSE control } & 3 & $80.80 *$ \\
\hline & 6 & 33.25 \\
\hline \multirow[t]{2}{*}{ Ryff autonomy } & 3 & 1.33 \\
\hline & 6 & 0.80 \\
\hline \multirow[t]{2}{*}{ Ryff self-acceptance } & 3 & -0.89 \\
\hline & 6 & -1.74 \\
\hline \multirow[t]{2}{*}{ Ryff environmental mastery } & 3 & -0.04 \\
\hline & 6 & -2.09 \\
\hline \multicolumn{3}{|c|}{ Post hoc analyses: self-management factor scores } \\
\hline \multicolumn{3}{|c|}{ Informant } \\
\hline \multirow[t]{2}{*}{ Behavioral involvement } & 3 & -0.01 \\
\hline & 6 & 0.26 \\
\hline \multirow[t]{2}{*}{ Cognitive involvement } & 3 & 0.31 \\
\hline & 6 & $0.75^{*}$ \\
\hline \multicolumn{3}{|l|}{ Patient } \\
\hline \multirow[t]{2}{*}{ Self-efficacy } & 3 & $0.35^{* *}$ \\
\hline & 6 & 0.26 \\
\hline \multirow[t]{2}{*}{ Well-being } & 3 & -0.02 \\
\hline & 6 & -0.36 \\
\hline
\end{tabular}




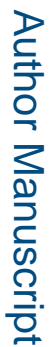

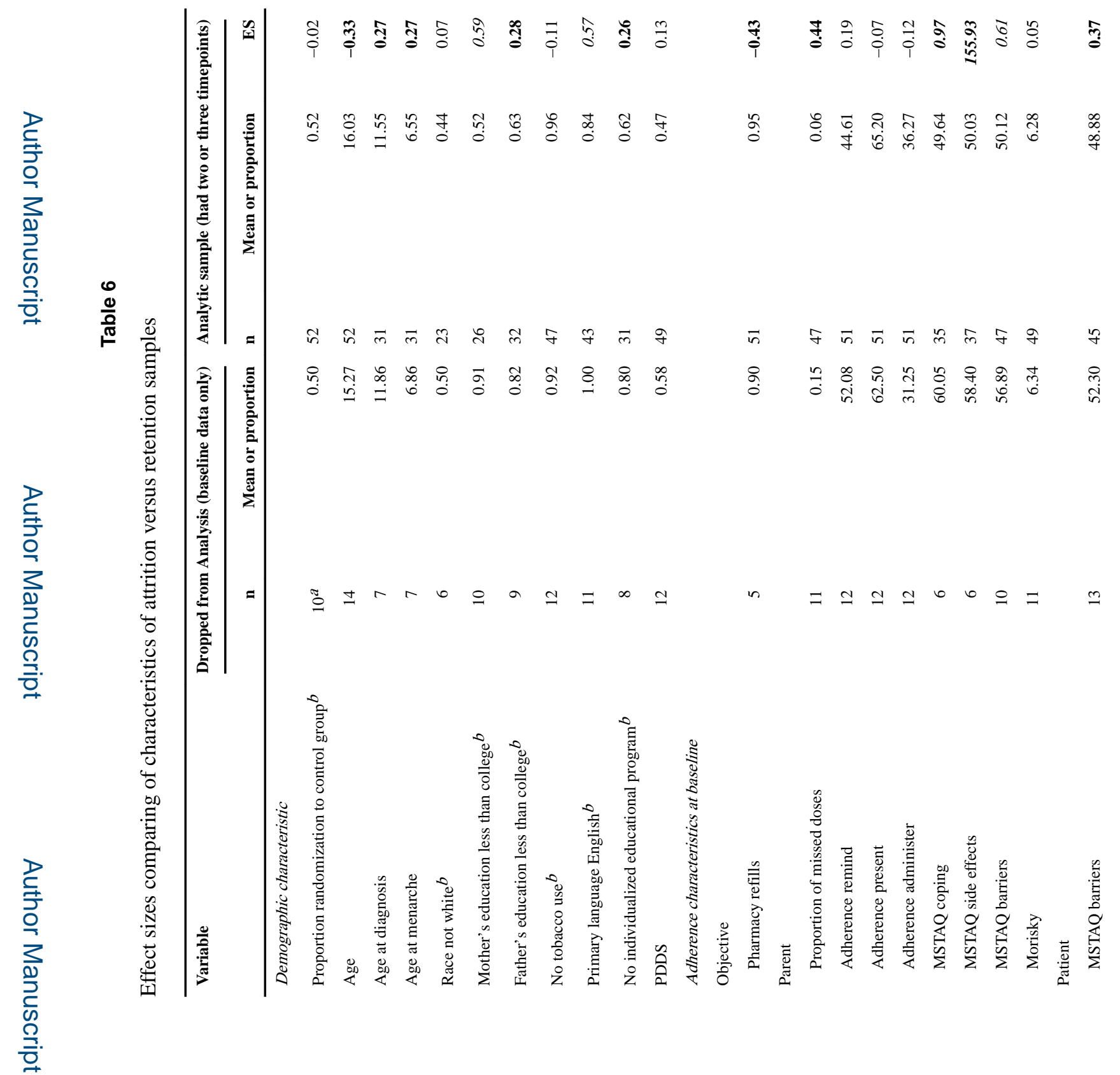

Qual Life Res. Author manuscript; available in PMC 2018 September 21. 
Yeh et al.

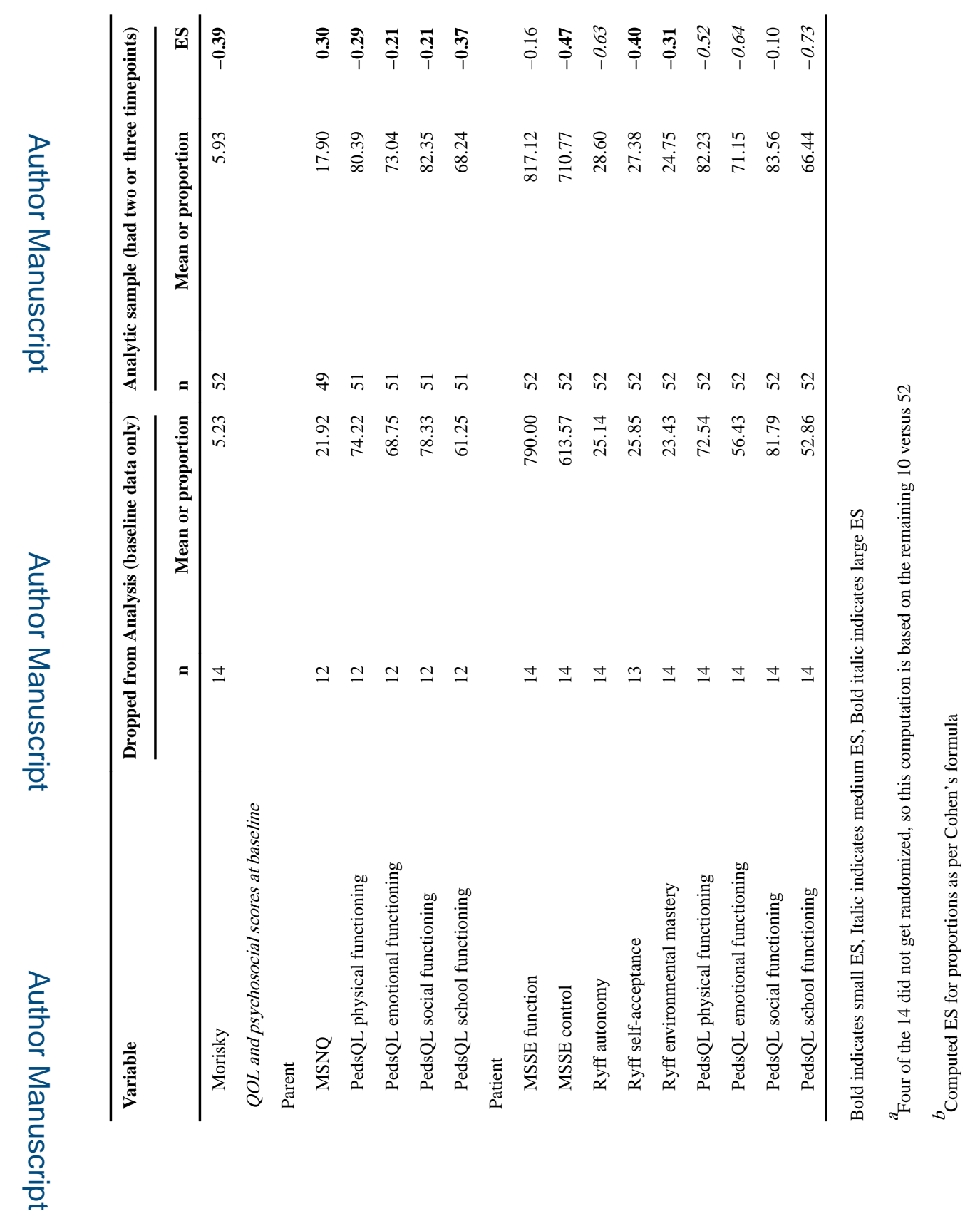

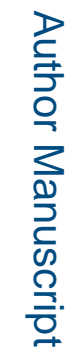

Qual Life Res. Author manuscript; available in PMC 2018 September 21. 


\section{Table 7}

Site investigators and study coordinators

\begin{tabular}{|c|c|c|}
\hline Site & Site Investigator & Site Coordinator(s) \\
\hline Alberta Children's Hospital, Calgary, AB & Dr. Jean K. Mah & Tiffany Haig/Karla Sanchez \\
\hline $\begin{array}{l}\text { Baylor College of Medicine, Texas Children's } \\
\text { Hospital, Houston, TX, USA }\end{array}$ & Dr. Tim Lotze & $\begin{array}{l}\text { Rubi Mendoza/Marija Stosic/Mariam Pontifes/ } \\
\text { Rory Mahabir }\end{array}$ \\
\hline Boston Children's Hospital, Boston, MA, USA & $\begin{array}{l}\text { Drs. Mark Gorman and Lauren } \\
\text { Mednick }\end{array}$ & Susana Camposano \\
\hline $\begin{array}{l}\text { Children's Hospital of Philadelphia, Perelman } \\
\text { School of Medicine, University of Pennsylvania, } \\
\text { Philadelphia, PA, USA }\end{array}$ & $\begin{array}{l}\text { Drs. Brenda Banwell and Amy } \\
\text { Waldman }\end{array}$ & Geraldine Liu/Amy Lavery/Maleka Smith \\
\hline $\begin{array}{l}\text { Hospital for Sick Children, Toronto, Ontario, } \\
\text { Canada }\end{array}$ & Dr. E. Ann Yeh & $\begin{array}{l}\text { Stephanie Grover/Austin Noguera/Carolynn } \\
\text { Darrell/Dr. Ruth Slater }\end{array}$ \\
\hline $\begin{array}{l}\text { University of Alabama Birmingham, Birmingham, } \\
\text { AL, USA }\end{array}$ & Dr. Jayne Ness & Sarah Dowdy \\
\hline $\begin{array}{l}\text { University of California San Francisco, San } \\
\text { Francisco, CA, USA }\end{array}$ & $\begin{array}{l}\text { Drs. Emmanuelle Waubant and } \\
\text { Jennifer Graves }\end{array}$ & Janace Hart/Hardeep Chohan \\
\hline University of Colorado Denver, Denver, CO, USA & Dr. Teri Schreiner & Alexander Stein/Kawonas Jenkins \\
\hline University of Pittsburgh, Pittsburgh, PA, USA & Dr. Gulay Alper & - \\
\hline
\end{tabular}

